Editorial

\title{
The impact of clinical research and evidence-based practice in occupational therapy ${ }^{1}$
}

\author{
Franklin Stein ${ }^{\mathrm{a}, \mathrm{b}}$ (D) \\ ${ }^{a}$ Editor, Annals of International Occupational Therapy, Thorofare, USA.

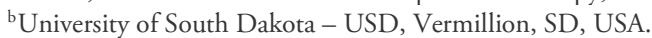

How to cite: Stein, F. (2020). The impact of clinical research and evidence-based practice in occupational therapy. Cadernos Brasileiros de Terapia Ocupacional. 28(1), 1-4. https://doi.org/10.4322/2526-8910.ctoED2801

Clinical research has been the engine for progress in the health and medical professions in the last 100 years and is the basis for improving the quality of client care. The important discoveries for example in anti-biotics, cancer treatment, stroke and cardiac rehabilitation have come about through research published in refereed journals. As editor of a journal in occupational therapy I judge the quality of a manuscript by its potential impact on improving clinical practice. In this short Editorial my objectives are to define how clinical research in occupational therapy can be is used in evidence based practice (EBP) and to give examples of potential research studies.

In a recent issue of the Annals of International Occupational Therapy (Annals of International Occupational Therapy, 2019) the following studies were published: Effects of Meditative Movements on the Symptoms of persons with Chronic Health Conditions; Pilot Study of an Occupational Therapy Dynavision D @ Protocol for Enhancement of Visual Performance among Collegiate Athletes; Occupational Therapy Interventions in Skilled Nursing Facilities: A Scoping Review and Effect of a Sound-Based Intervention on a 7-Year -Old Child Diagnosed with Auditory Processing Disorder. In all of the above studies there is an identified intervention such as meditation, Dynavision D2, occupational therapy in a skilled nursing home and sound-based protocol.

The title in a research study should clearly identify the client population such as in the above studies, persons with chronic health conditions, collegiate athletes, nursing home residents and a child with a auditory disability. The results of these research studies form the basis for evidence-based practice in that they can be used to improve clinical practice.

First of all, how is research best defined? What is the relationship between research and evidence-based practice in occupational therapy? How are the results of research transferred to occupational therapy practice by using evidence based practice (EBP)? What are examples of potential research studies in occupational therapy?

Research in general can be defined as a systematic and objective investigation through identifying a significant problem; stating a testable hypothesis or guiding question, and; objectively collecting primary data. Applying research to clinical

${ }^{1}$ Portions of this Editorial is from the recent book Clinical research in Occupational Therapy (Rice et al., 2019). 
practice in occupational therapy can be defined as the systematic and objective study of interventions to improve health, prevent illness and develop or restore function in individuals with disabilities.

EBP refers to applying the results of research to occupational therapy practice from a systematic review of research or a meta-analysis where data is statistically combined. Evidence-based practice as initially defined by Sackett et al. (1996, p. 71), is: "The conscientious, explicit and judicious use of current best evidence in making decisions about the care of the individual patient. It means integrating individual clinical expertise with the best available external clinical evidence from systematic research".

In applying EBP to occupational therapy the practitioner in a way has to evaluate the evidence and determine how a specific clinical intervention can be applied to a specific client. Research in general is a cumulative process where the evidence is continually evaluated and refined through replication. For example, if one research study shows that cognitive behavior therapy (CBT) is effective in reducing the symptoms of depression there is still a need to replicate the study with other populations so as to generalize the results. The refinement of the results also comes with standardizing the intervention protocol and in determining which types of clients will benefit most from CBT. What are the components of a research study related to EBP?

- Is the title representative of the manuscript? For example, is the independent variable identified such as the intervention. Is the dependent variable identified such as the desired outcome? Is the population sample identified? Is the setting for the study identified? For example, a clinical research study could be titled: The effects of constraint therapy (Intervention) on functional abilities (outcome) in patients with stroke (sample population) in a rehabilitation (setting);

- Does the abstract summarize the major points in the study? The abstract should include the objective of the study such as to evaluate the effectiveness of an intervention with a specific population. Are the methods for collecting data included such as screening criteria for subject selection, assessments used to measure outcome and the design of the study including control group. The next section of an abstract includes the results that are stated concisely and the conclusions which include the recommendations for further research and possible limitations of the study;

- Is there justification for the significance and need for study? The author should introduce the study by citing statistics that demonstrate the significance and need for carrying out a clinical research study such as the incidence and prevalence of stroke;

- Is the literature review current and comprehensive? The literature cited should demonstrate that the author has done an extensive review of clinical studies that are current and come from a wide source of journals. Pubmed.com is a good source initially for identifying research studies in occupational therapy;

- Does the study have internal validity (control of extraneous variables)? In the methodology section the author describes in enough detail the procedure used in collecting data. The methods should be operationally defined so that the study can be replicated. The procedure for screening subjects such as inclusion criteria is 
included in this section. The assessments for measuring outcome should include reliability and validity data;

- Is the results section objectively presented by the author? Are there tables of data to show statistical analyses of the data?

- In the discussion section does the researcher compare the current results with the reported results from the literature?

- Are the conclusions consistent with the results? Does the author state the limitations of the study and recommendations for further research?

In applying the concept of EBP to occupational therapy, clinical reasoning is the most important skill necessary in transferring research results to clinical practice. The occupational therapist has to weigh the validity of the research study and its relevance to practice. In general, the process of applying EBP to occupational therapy includes:

1. Evaluating the quality of a specific intervention such as research on constraint therapy in stroke rehabilitation;

2. Creating a data base for teaching, for example by using meta-analysis to determine the best interventions for children with autism;

3. Integrating recent research evidence with current practices in occupational therapy and by so doing improving and refining clinical practice;

4. Facilitating the transfer of knowledge from research to practice.

\section{What are Examples of "best evidence" in Occupational Therapy}

Clinical research provides best evidence for clinical practice such as: randomized control trials (RCTS) are based on a standardized treatment protocol (intervention) that can be replicated by other researchers, standardized outcome measures that have good reliability and validity and a specific population that is operationally defined. The methodology involves a pretest-posttest design with a control group.

Another research design involves prospective studies of single subjects which are case studies that are pilot projects where the results can be used to generate further research. Occupational therapists who work directly with clients can design experimental case studies by using a pre-post design and standardized measurements. These case studies can be replicated and refined.

Retrospective study of a single subject is another method for understanding how a client responded to an intervention. For example, clients who are successful in controlling an addiction can be compared to another client who has continual relapses. What are the factors retrospectively in the success or failure of the interventions?

Longitudinal or follow-up studies of interventions can help us understand the dynamics and long term effects of interventions such as in smoking cessation programs.

\section{What are the Skills Needed to Utilize EBP?}

- A critical attitude towards one's own clinical practice; 
- Intellectual curiosity in staying current with the published literature;

- Knowledge of research methodology and statistics;

- Familiarity with pubmed.gov and Cochrane Reviews for retrieving research studies;

- Ability to systematically without bias to analyze the published literature;

- Ability to transfer research data to clinical practice.

\section{What are Potential Areas for Clinical Research in Occupational Therapy}

- Applying ergonomics in adapting home environments for those with disabilities;

- Evaluating purposeful and meaningful activities in treating individuals with mental illness;

- Developing standardized teaching activities of daily living to individuals with disabilities;

- Is sensory integration effective with children diagnosed with autism?

- Developing splints for individuals with hand disorders;

- Adapting a wheelchair for efficient use in individuals with spinal cord injuries;

- Evaluating and assisting individuals with disabilities to drive a car;

- Improving hand writing skills in children with learning disabilities;

- Applying constraint-induced movement therapy with individuals with hemiparesis;

- Improving cognitive function in individuals with dementia;

- Assisting individuals with multiple sclerosis to work in paid employment;

- Applying stress management programs for individuals with mental illness;

- Using biofeedback in interventions for decreasing incontinence;

- Developing prescriptive exercise programs for post cardiac patients;

- Evaluating sensory stimulation programs for infants in neonatal units;

- Teaching how to use prosthetic devices.

In conclusion, clinical research in occupational therapy in tandem with evidencebased practice can have a powerful effect in improving the quality of patient care and provide evidence for using interventions.

\section{References}

Annals of International Occupational Therapy. (2019). 2(2). Thorofare: Healio. Retrieved in 2019, October 31, https://www.healio.com/occupational-therapy/journals/otannals/2019-4-2-2

Rice, M. S., Stein, F., \& Tomlin, G. (2019). Clinical research in occupational therapy. Thorofare: SLACK Inc..

Sackett, D. L., Rosenberg, W. M., Gray, J. A., Haynes, R. B., \& Richardson, W. S. (1996). Evidencebased medicine: what it is and what it isn't. British Medical Journal, 312(7023), 71-72. PMid:8555924. http://dx.doi.org/10.1136/bmj.312.7023.71. 